\title{
Evaluation of the aerodynamic performance increase thanks to a morphing A320 wing with high-lift flap by means of CFD Hi-Fi approaches
}

\author{
A. Marouf* and Y. Hoarau ${ }^{\dagger}$ \\ ICUBE, Strasbourg University France \\ J.B. Vos ${ }^{\ddagger}$ and D. Charbonnier ${ }^{\S}$ \\ CFS Engineering, EPFL Innovation Park, Batiment A, CH-1015 Lausanne, Switzerland \\ Y. Bmegaptche Tekap "I and M. Braza " \\ Institut de Mécanique des Fluides de Toulouse (IMFT), France
}

\begin{abstract}
The flow around a two-element large scale wing-flap configuration with a dynamic morphing of the flap's trailing-edge is investigated by means of two dimensional and three-dimensional high-fidelity numerical simulation. A detailed explanation is provided for the implemented methods to compute the time depend displacement and the dynamic grid deformation in the NSMB code. The aim of this study is to simulate the morphing of the flap's trailing-edge at high frequency and low amplitude actuations. The morphing introduces new small anticlockwise vortices in the wake at high frequency. This effects are also observed in TR-PIV experimental results. This technique creates an eddy blocking effect in the wake shear-layer, which leads to a breakdown of the coherent structures in the wake, make a thinner wake and decrease the aerodynamic noise. Several unsteady simulations were carried to find out the optimal frequencies and amplitudes allowing an increase of the aerodynamic performances as the lift force and decrease the drag.
\end{abstract}

\section{Nomenclature}

$\begin{array}{ll}a & =\text { amplitude of actuation } \\ C_{L} & =\text { lift coefficient } \\ C_{D} & =\text { drag coefficient } \\ f_{a} & =\text { frequency of actuation } \\ C & =\text { total chord } \\ C_{\text {flap }} & =\text { chord of the flap } \\ \mathrm{d} t & =\text { time step } \\ d & =\text { morphing disctance } \\ T u & =\text { turbulence intensity } \\ \alpha & =\text { angle of incidence } \\ \delta & =\text { angle of deflection } \\ C P & =\text { pressure coefficient } \\ M & =\text { Mach number } \\ R e & =\text { Reynolds number }\end{array}$

*PhD Student, ICUBE, Strasbourg University France, amarouf@ unistra.fr

†Professor, ICUBE, Strasbourg University, hoarau@unistra.fr

† Director, CFS Engineering,jan.vos@cfse.ch, Senior AIAA member

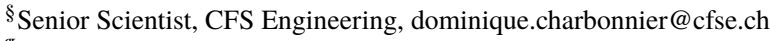

IPhD Student, Institut de Mécanique des Fluides de Toulouse IMFT, yannick.bmegaptchetekap@imft.fr

"Research Director, Institute de Mécanique des Fluides Toulouse (IMFT), marianna.braza@imft.fr 


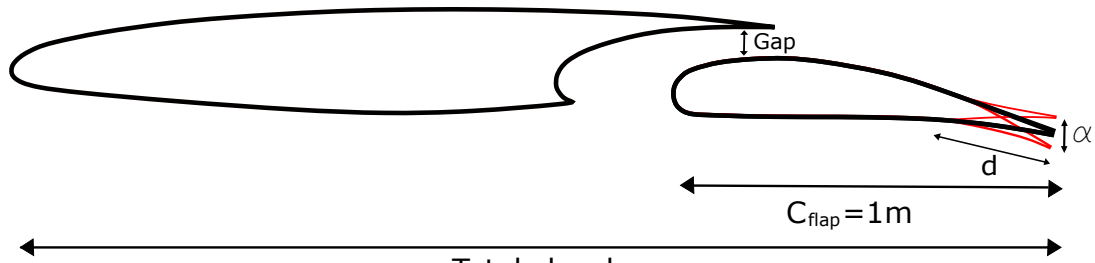

Total chord

Fig. 1 Schematic of the Large-Scale A320 wing-flap configuration and the trailing-edge dynamic deformation with high frequency low amplitude actuations

\begin{abstract}
II. Introduction
THIs study is part of the electroactive morphing activities in the context of the SMS (Smart Morphing and Sensing)

European project (www.smartmorphing.org/SMS). 2D and 3D numerical simulations for the hybrid electroactive morphing operating at different frequencies, amplitudes and camber will be presented. The study includes high-fidelity simulations of a Large Scale prototype for High lift two element wing-flap Airbus A320 at the takeoff position. The wing has a total chord of $3 / 4$ and the flap $1 / 1$ of the real scale. The flap geometry is the same as the real A320. The vibration of the trailing edge interacts with the fluid flow and modifies the turbulent structures in the wake which can lead to the breakdown of the coherent structures at low frequency. A considerable number of tests have been done to find the optimal frequency of the near trailing edge vibration, the amplitude of the corresponding slight deformation and the camber (deflection of the flap) to increase wing performances as an increase of the lift and suppression of the wake instabilities by attenuating the predominant frequency of the shear layer instabilities.
\end{abstract}

Most of the works in literature are based on the static morphing, static cambering and flap deflection targeting the optimisation of the aerodynamic forces. However, dynamics morphing (time-depend shape deformation) is not deeply investigated by researcher in the numerical field and could have an impact over the dynamic behaviour of the wake instabilities and the aerodynamic noise. As for instance, M. Chinaud et al 2014 [1] studied experimentally electroactive morphing effects obtained by Shape Memory Alloys (SMA) for the bending of a flat plate at $10^{\circ}$ of incidence and high Reynolds number $(200,000)$ at low speed by means of the PIV. This actuation at low frequency modifies the turbulent structures and the instabilities in the near wake. The shear-layer amplitude is attenuated and of the spectral energy with a cambering of the plate. Furthermore, J. Scheller et al [2] performed experimentation of the trailing edge morphing of a NACA wing using TRPIV (Time Resolved PIV) to post-treat the time depend results showed a considerable reduction of the components of the Reynolds stress and a simultaneous significant diminution of the predominant natural frequency in the wake. The association of low-frequency high-deformation is the results of a multidisciplinary collaboration between the two Laboratories Institut de Mécanique des Fluides de Toulouse (IMFT) and Laboratoire Plasma et Conversion d'Energie (LAPLACE) and reported as 'hybrid electroactive morphing', able to operate at different time and length scales of the turbulent motion [3]. In addition, G. Jodin et al [4] performed experimentation of an A320 morphing prototype of $70 \mathrm{~cm}$ chord, able to camber of $15 \%$ of the total chord by means of Smart Memory Alloy (SMA) cambering at low frequencies less than $1 \mathrm{~Hz}$ and equipped by MFC (Macro-Fiber Composite) piezo-actuators in the near trailing-edge of the wing to vibrate from 30 to $500 \mathrm{~Hz}$, it is called hybrid electroactive morphing. G. Jodin et al [4] measured the pressure and aerodynamic forces measurements and revealed that the association of both classes of actuations was able to produce an order of $3 \%$ more in the lift forces, than the only use of the camber control.

Researchers in NASA have successfully made an Elasticity shape Air Vehicle Project [5] [6] and revealed an enhancement of the aerodynamic performances, which results in drag reduction during the cruise and increasing of the lift during take-off and landing. The pocket that generate high vorticity and aerodynamic noise is defined as the gap and presented in the Fig. 11 which separates the flap and the wing, at a certain size of the gap, the flap is decoupled from the wing which may induce a loss in $C L / C D$ ratio. Recently J.B. Tô et al (2019) [7] studied numerically the effect of static and dynamic morphing of a reduced prototype of an A320 wing at a transonic speed. The morphing induces a lock-in of the buffet's frequency with the actuating frequency, reduces the separated region of the boundary layer and the thinning of the wake's width. In addition, a slight deflection of the trailing-edge upward showed efficient lift to drag ratio compared to the classical fixed wing-flap.

This study focuses on the understanding of the morphing effects on the flow around the two-element wing-flap system and the improvement of the aerodynamic forces in the take off position of a two-element wing flap of the Airbus A320 at a large scale. The objectives of the present paper are summarized as follows : 

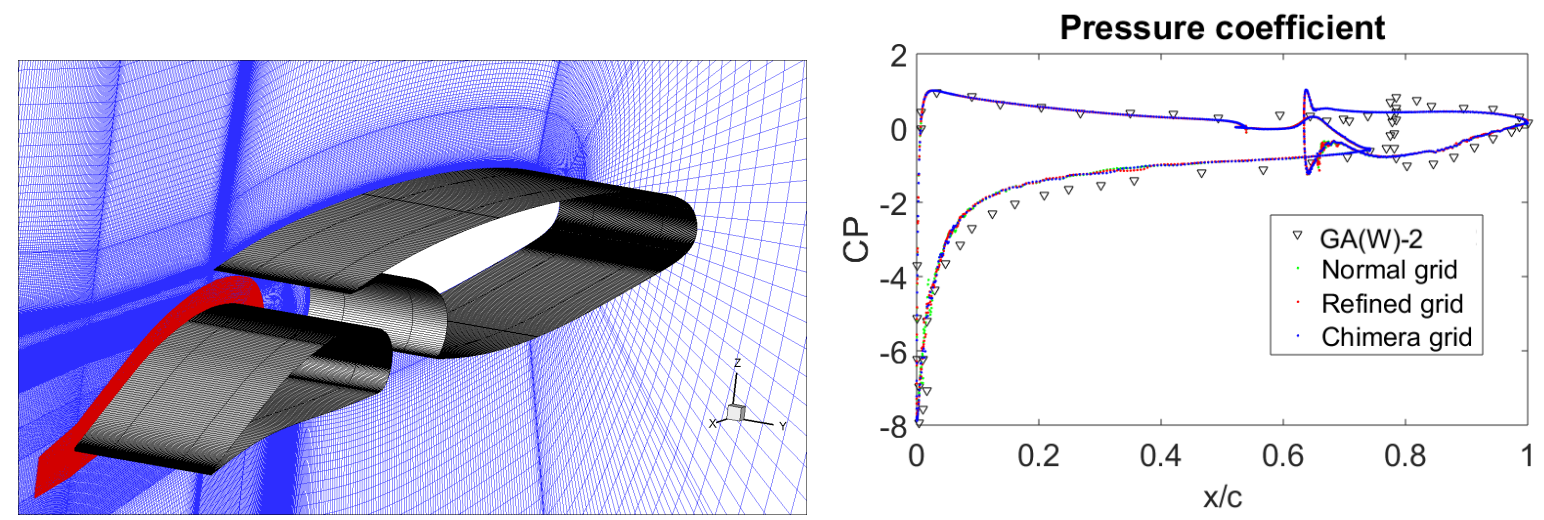

Fig. 2 Left : The two-element wing-flap Chimera (overset) mesh used for test validation. Right : Pressure coefficient for different grids and GA(W) 2 element airfoil-flap

- Explain the dynamic deformation of the structured multi-block grid using advanced numerical methods implemented in the NSMB code [8] with a conservation of the same mesh properties.

- Examine the main physical flow around the two-element configuration and reveal the existence of different coherent structures in the wake.

- Track the flow modification and feedback of the dynamic time depend morphing of the flap's trailing-edge.

- Identify the optimal frequency-amplitude region to obtain better aerodynamic performances

\section{Numerical methods and turbulence modelling}

\section{A. Flow regime}

Concerning the design of the Large Scale (LS) A320 two-element wing-flap prototype, In the context of the SMS project the scale of the Airbus A320 wing was modified due to a limitation of the IMFT wind tunnel. The wing's chord is taken $2 / 3$ of the real Airbus wing. However, to analyse the real effects of the morphing, the flap chord corresponds to the real flap of the A320. The physical configuration of the flow is taken as a subsonic at a free-stream boundary conditions. A low Mach number $M=0.032$ and a Reynolds number of $R e=2.25$ million have been chosen. A high angle of attack is chosen $\alpha=8.2^{\circ}$ which corresponds to the range of angles in the Take-off position seen in literature. The flap is deflected with $\delta=10^{\circ}$ from its initial position. Two dimensional and three-dimensional simulations were performed for the static case (no vibrations) and taken as reference to compare with the dynamic morphing at high-frequency low-amplitude configuration. Optimal frequencies of vibration varies in the range of $f_{a}=[60-400]$ $\mathrm{Hz}$ with a fixed vertical actuation of $0.7 \mathrm{~mm}$. The morphing revealed through the previous studies an enhancement of the aerodynamic performances and a suppression of the wake instabilities compared to the classical fixed system. The flap's trailing-edge is deformed dynamically in experimental setup using the Electro-Mechanical Actuators (EMA) to achieve higher mechanical efficiency, increase the loading capacity and the speed operation which is under construction. In the numerical simulations an implementation of an imposed deformation and automatic remeshing methods are used and will be presented in the next section.

\section{B. Numerical parameters}

A CH topology for the grid generation was selected for this study to simulate takeoff near to the real conditions. An overset mesh is used to validate the results. This method is a powerful tool to simulate complex fluid problems, not every geometry can be well represented using a single continuous mesh, different geometrical features as the wing and the flap are better represented by different mesh types. Preparing this type of mesh is a time consuming and complicated. Overset allows us to construct a structured domain between the airfoil and the flap, each grid being optimized to the component and its role in the simulation. A normal and finer Chimera (overset) grid and non-Chimera (single) multi-block grids built in our research team are used in this work. The Chimera grid contains two independent 


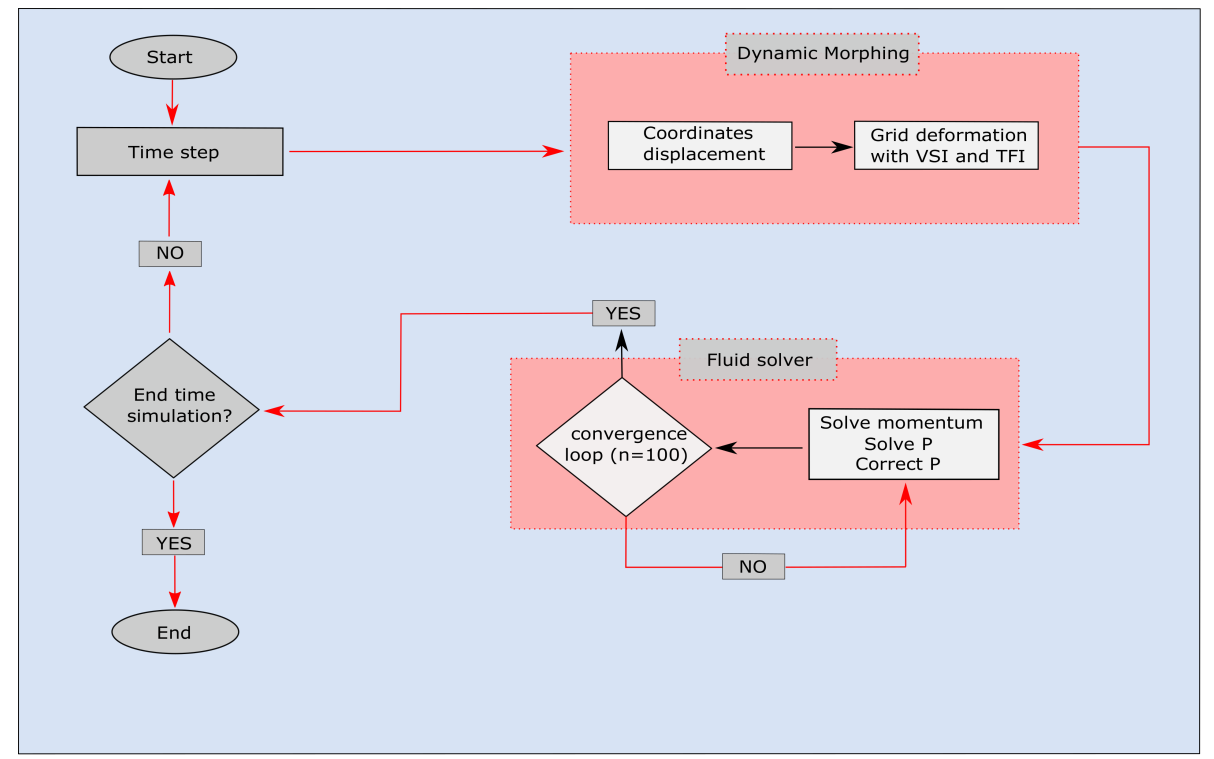

Fig. 3 Flow chart of the dynamic morphing implemented in the NSMB solver

grids, the first grid is the fluid domain including the wing and the second one is around the flap in Fig. 2 (left). This grid is used for optional configurations of Take-off and Landing where the flap changes its deflection angle from $10^{\circ}$ to $40^{\circ}$ degrees. The grids have been tested in the NSMB code and a mesh independence have been studies for the pressure coefficients with the experimental results is provided in Fig. 2 (right). The results show a very similar Pressure coefficient $C p$ for both grids. The refined single grid is selected to continue this study. The presence of the walls in the IMFT wind tunnel S4 has an effect on the pressure coefficient, the wing was mounted wall-to-wall in the wind tunnel and a good comparison of numerical experimental will be achieved in the future. We compared for this present study the flow in 2D in the Fig. 2 (right) the numerical simulation to the results of GA(w)- 2 airfoil-flap (W. LU [9]) which is almost similar airfoil properties as the A320, but with a smaller flap.

The simulations have been carried out with the NSMB solver. The NSMB solver is the fruit of a European consortium that included Airbus from the beginning of ' 90 s, as well as main European aeronautics research Institutes, as KTH, EPFL, IMFT, ICUBE, CERFACS, Univ. of Karlsruhe, ETH-Ecole Polytechnique de Zurich, among other. This consortium is coordinated by CFS Engineering in Lausanne, Switzerland. NSMB is a structured code that includes a variety of efficient high order numerical schemes and of turbulence modelling closures in the context of LES, URANS and of hybrid turbulence modelling. A first description of the code can be found in Vos et al. (1998) [8] concerning the versions of this code in the decade of '90s. Since then, NSMB highly evolved up to now and includes an ensemble of the most efficient CFD methods, as well as adapted fluid-structure coupling for moving and deformable structures. These developments can be found in Hoarau (2002) [10] and Hoarau et al (2016) [11] regarding URANS modelling for strongly detached flows, Martinat et al. (2008) [12], in the area of moving body configurations, Barbut et al. (2010) [13] and Grossi et al. (2014) [14] allowing for Detached Eddy Simulation with the NSMB code. NSMB solves the compressible Navier-Stokes equations using a finite-volume formulation on multi-block structured grids.

In the present work, a fourth-order central scheme is used for the spatial scheme and an upwind second order for the temporal scheme. Dual-time stepping for the temporal discretization is chosen. Furthermore, artificial compressibility for the preconditioning is used to simulate the incompressible flow at low Mach speed range. After a detailed investigation a physical time step of order $10^{-5} \mathrm{~s}$ is selected for $2 \mathrm{D}$ simulations for 100 inner iterations for the process of convergence each time step.

\section{Turbulence modelling}

The unsteady turbulent flow around the Airbus two-element wing-flap at high Reynolds and angle of incidence is predicted by the Organized Eddy Simulation turbulence model [15] [16]. Reinforcement of the turbulent structures with a modification of the $C_{\mu}=0.03$ has an impact over the non-equilibrium turbulence near the wall regions of the Boundary Layer (BL). Turbulent length scale used in the Detached Eddy Simulation has been adopted for the OES model. In 

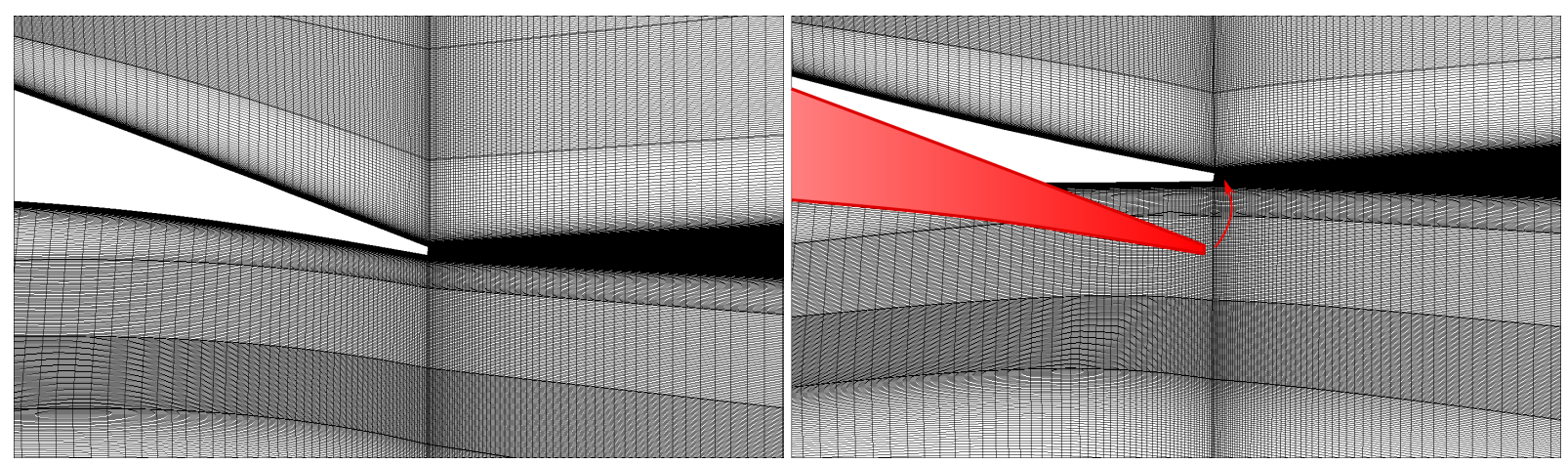

Fig. 4 Left : A Zoom at the Static grid of the A320 near to the flap's trailing edge. Right : an upward dynamic deformation and the (red) refers to the original position

addition, this model provides an efficient and accurate Boundary Layer BL detachment compared to the standard $K-\epsilon$ model for configurations at high Reynolds number of the order of $10^{6}$ and captures low frequency modes as von kármń instability, flutter and buffet phenomenons. This OES approach splits the spectrum of energy into two parts. The first one groups the organised flow turbulent structures which is resolved and the second one consists of the chaotic process of the random turbulence which are modelled. However, the Unsteady RANS models such as the standard $K-\epsilon$ tested in our case are not efficient.

\section{OES-DDES}

Three-dimensional simulations were performed to analyse the flow dynamics through the spanwise direction for the two-element wing-flap configuration. The grid is fine enough to capture the flow instabilities, resolve a significant part of the turbulence generated by the high-frequency low-deformation dynamic morphing effects near the trailing-edge region.

Multi-block structured mesh of around $30 \times 10^{6}$ cells generated in our team and composed of 40 cells through the spanwise direction over $0.11 C$, which considered sufficient to capture three-dimensionality. Delayed Detached Eddy Simulation (DDES) model [17] grid has a homogenous grid cells $\Delta z \approx \Delta x$ and the OES-DDES model is selected for this study. The model switches from RANS to LES region by means of the $F_{d}$ coefficient. For the URANS model the OES was considered as a good model to capture the unsteadiness and the flow detachment near to the boundary. The classical Smagorinsky model is selected for the LES model and based on the subgrid length-scale. Moreover, the transient simulation time step is $5 \times 10^{-6} \mathrm{~s}$ which allows obtaining better convergence and capture smaller length scale turbulent structures.

\section{E. Morphing strategy}

Unsteady flow calculations for problems with deformable surface shapes is investigated using grid deformation for a multi-block, structured mesh. A polynomial second order equation is implemented in the NSMB code to calculate the time-dependant dynamic displacement. A frequency $f_{a}$ of actuation, an amplitude $a$ and a displacement $d$ are imposed for the the flap's trailing-edge as presented in the Fig. 1. The Figure 4 represents an example of actuation compared to the static case. This dynamic vibration modifies the flow in the shear-layer of the wake and changes the behaviour of the flow dynamics. Fast and reliable method for deforming the computational grid during each step of the time simulation is needed while maintaining a good mesh quality as the orthogonality and the smoothness. The remeshing algorithm implemented in the code NSMB combining the Volume Spline Interpolation (VSI) and Transfinite Interpolation (TFI) [18]. These methods collect at first the imposed displacement of the trailing-edge and creates a list of vortices. The Volume Spline Coefficients calculation requires a matrix inversion of a full square matrix $N+1$ rows where $N$ is the number of vertices on the configuration surface. Only moved block edges using VSI method are taken into account which is one of the advantages of the VSI method that does not require all the coordinates. Next, the Trans Finite Interpolation is used to generate the displacement of block faces coordinates in two-dimension or the volume in three-dimension. Finally, the new deformed grid is computed based on the imposed deformation fixed as an input as presented in the Fig. 4 which shows the new computed grid after a deformation. These methods are used with a great 

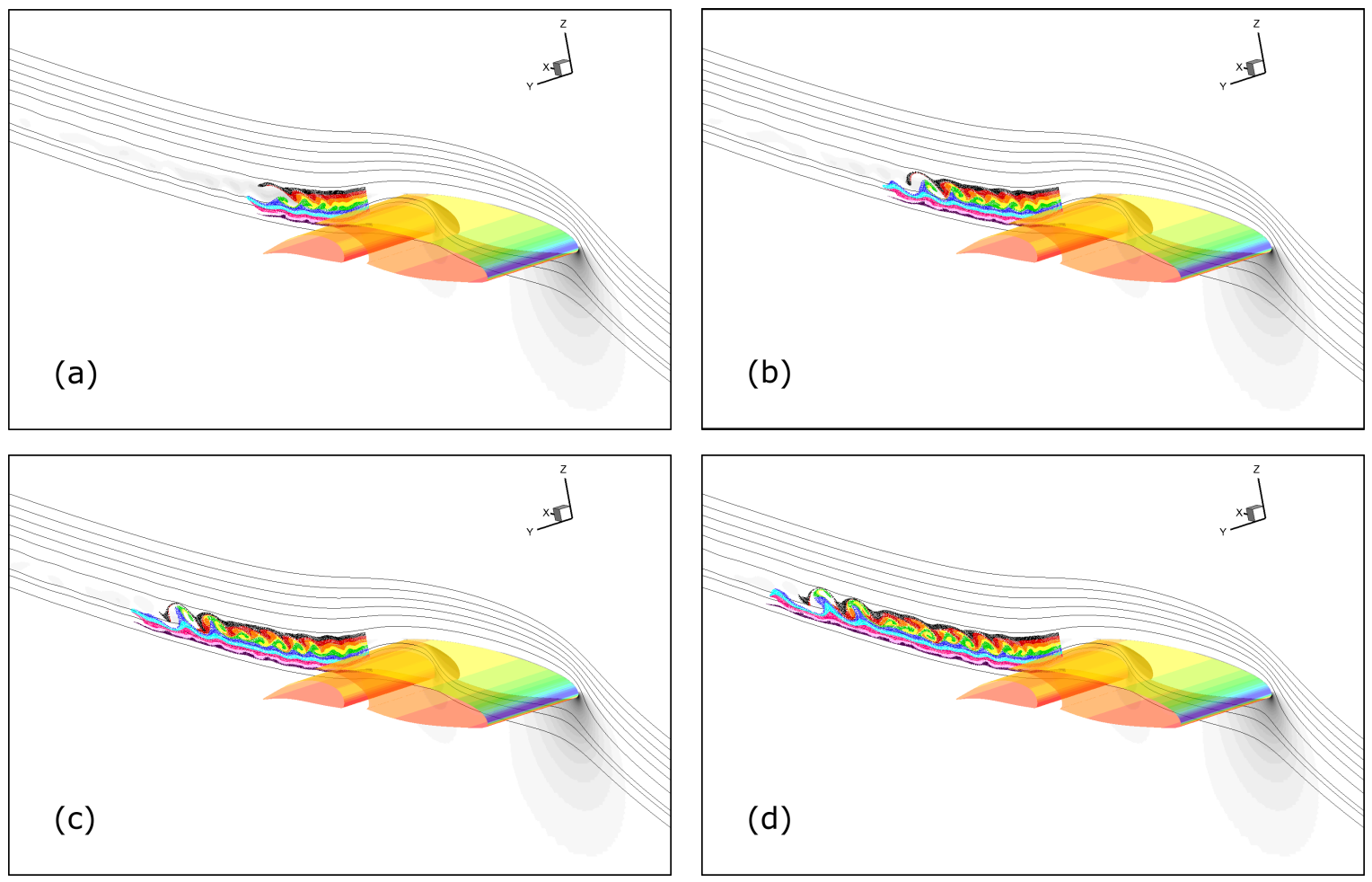

Fig. 5 Streaklines technique visualization injected at the end of the flap showing the appearance of the wake instabilities for different time simulation

success and they are able to compute high-quality mesh deformation allowing preservation of a good orthogonality. In addition, the TFI method is very fast since the computational effort and the number of volume grid points $N_{v}$ are proportional.

\section{Results}

According to the Organized Eddy Simulation method, the resolved part in the frequency analysis domain corresponds to the organised coherent parts of the flow as the shear layer instabilities, Kelvin Helmholtz (KH) and von kármán (vK) vortices. The Figure 5 represents the pressure distribution over the wing and several layers of calculated Streaklines injected in the wake at a fixed vertical line placed at $80 \%$ of the flap's chord system near to the trailing-edge. This technique helps to reveal the coherent parts existing in the wake. In addition, 1000 snapshots were saved each $10^{-3} \mathrm{~s}$ to capture the highest predominant frequency that exists in the wake. The Power Spectral Density method helps to reveal the formation of the $\mathrm{KH}$ vortices in the shear-layer in the near wake and their predominant natural frequency. These vortices expand and roll up to a von kármán (vK) vortices in the far wake. The presence of a large gap splits the wake flow into many parts. At the first, the wing region produces a large velocity deficit in the wake due to the high angle of incidence and the wing's chord of $2.4 \mathrm{~m}$. Then, the gap region separates both flows coming from the wing and the flap. The calculated mean streamwise velocity is not modified in this region. Finally, the flap flow presents less shearing compared to the wing, due to the new modified angle of incidence of the flap due to the presence of the wing and the flap's chord. A non-linear interaction exists between the wing and the flap shear-layers in the wake region. In this numerical study the von Kármán vortices are not predominant. However, the flow contains their signature further in the wake. By means of the PSD with the Welch's weighted overlapped segment averaging estimator [19] a predominant natural frequency in a fixed monitor point at $x / C=1.25$ behind the wing-flap system and inside the wake, within the order of $30 \mathrm{~Hz}$ and corresponds the cross of $\mathrm{KH}$ vortices in this point.

Understanding the vortex shedding phenomenon helps to control them through the morphing. The flap's trailing edge dynamic deformation (with vibration) introduces new turbulence structures in the wake that can produce an eddy blocking effect that could lead to a change in the wake behaviour. In the following section the dynamic effects on the 

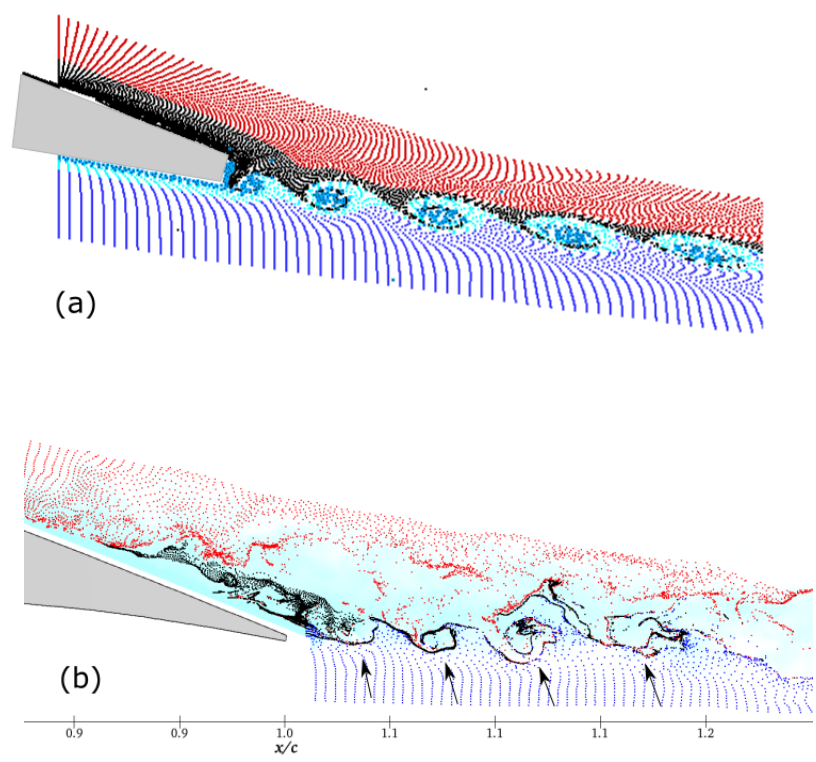

Fig. 6 Morphing Streakline technique visualization. $(a)$ : Numerical simulation of the two-element wing-flap A320 with actuation frequency $f_{a}=300 \mathrm{~Hz}$. $(b)$ : Experimental TR-PIV [20] of a single wing reduced scale A320 with an actuation frequency $f_{a}=220 \mathrm{~Hz}$

two-element wing-flap is treated and discussed.

\section{Effects of the dynamic morphing}

The objective of this section is to investigate the flow around the wing-flap system with numerical simulations and consists on the exploration of the frequency and of the amplitude trailing-edge vibrations effects and feedback. The wake mechanism and coherent structures are analysed by means of the OES model and their modification when the dynamic vibrations of the trailing-edge is applied. Forces measurements are carried out with mean values of the numerical signal of the lift and drag forces.

\section{A. Flow dynamics}

Calculated streaklines are useful to understand the real flow dynamics in the wake and its modification. 2D numerical results are compared to 3D experimentation of TR-PIV performed by [20] at a Reynolds number of 5. $10^{5}$ and angle of incidence of $10^{\circ}$ for a reduced scale prototype on the clean (one element) configuration of chord $c=0.7 \mathrm{~m}$. These experimentation were carried out with a morphing frequency of $220 \mathrm{~Hz}$ for a small actuation amplitude. Knowing that the numerical and the experimental configurations are not the same but surprisingly with the vibration a good consistency of the morphing is obtained between both results. Different colors were chosen to identify the wake structures, the particles colored in blue are placed in the pressure side (intrados) of the wing, the red particles in the suction side (extrados), these two layers are separated with black streaklines near to the trailing-edge and the separation of the boundary layer. Effects the morphing are visible, the vibration introduces constantly new smaller spaced vortices in both configurations presented in Fig. 6. The arrows in the Fig. 6p show the re-circulation of the black particles which is highly visible also in the Fig. 6a. The 2D simulations captures the coherent structures and filters smaller scale structures, on the other hand, in the experimentation, three-dimensional interaction and TR-PIV captures all the physics with smaller and larger turbulent structures. However, morphing effects are visible and these new vortices separate the lower and the upper shear-layer and present an eddy blocking effect as described by [21].

The Fig. 7 presents the static and the morphing configurations for the three-dimensional simulation by means of the OES-DDES hybrid model. The Q criterion fixed at the value of 1000 and colored by the Mach contours represents 

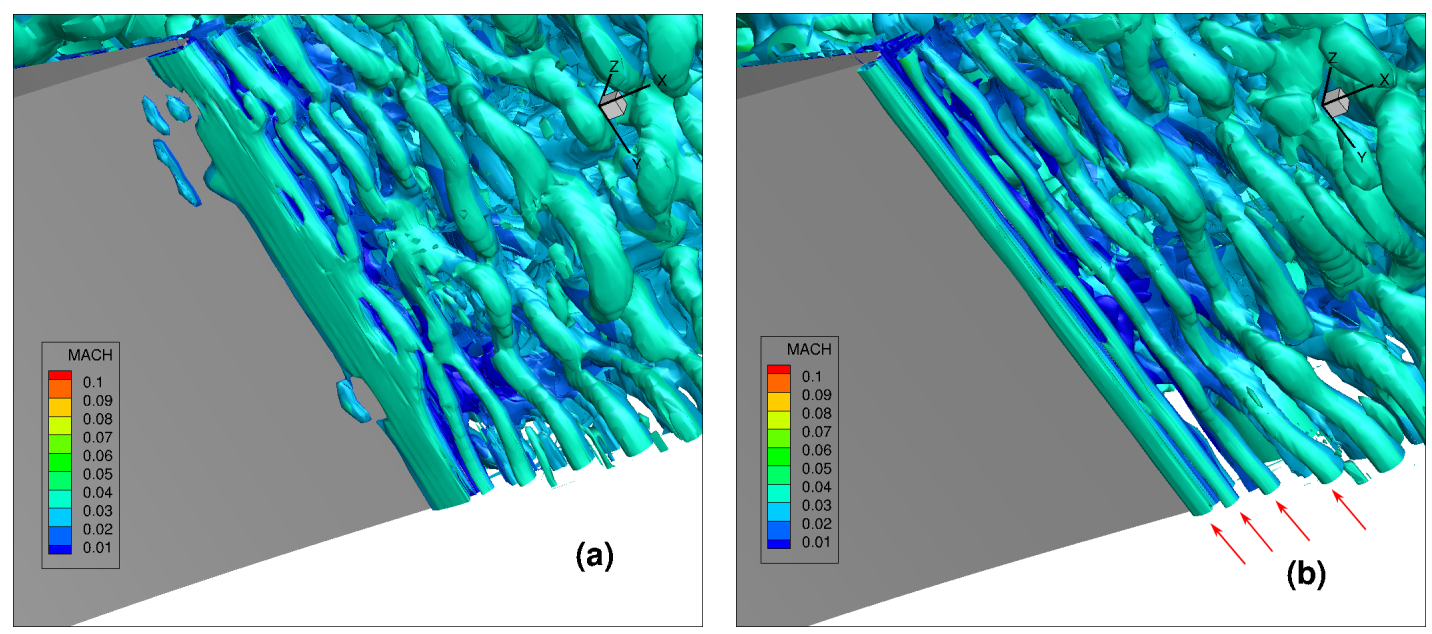

Fig. 7 Q criterion (1000) colored by the Mach contours under the flap's trailing-edge with the OES-DDES model in 3D simulation. (a) : Static case. $(b)$ : Morphing case

Table 1 Aerodynamic coefficients of the morphing configuration for difference frequency actuations

\begin{tabular}{|c|c|c|c|c|c|}
\hline Frequency of actuation $(\mathrm{Hz})$ & 60 & 100 & 200 & 300 & 400 \\
\hline$\frac{\left(C D-C D_{\text {static }}\right)}{C D_{\text {static }}} \times 100$ & -1.7971 & +0.7532 & +1.0664 & +2.8859 & +1.1857 \\
\hline$\frac{\left(C L-C L_{\text {static }}\right)}{C L_{\text {static }}} \times 100$ & -0.2918 & +0.5797 & +0.3465 & +0.5529 & +0.2557 \\
\hline
\end{tabular}

the three-dimensionality in the span wise in the Fig. 7 near the flap's trailing-edge. The spanwise velocity is amplified when the Reynolds number is higher, this leads to a breakdown of the vortices rows and appearance of the three-dimensional structures. In addition, a pressure gradient in the $x$ direction creates a vortex dislocation in the spanwise direction. However, the high-frequency low-deformation morphing modifies the flow behaviour in the same region. The uniform vibration introduces rows of anticlockwise vortices as presented in the Fig. $7 \mathrm{~b}$ which are dissipated over $0.2 C$ approximately. These results fit with two-dimensional simulations as presented in the previous sections.

The generated rows interact with the shear-layer and create an eddy blocking effect, which helps to make a thinner wake, decreases the aerodynamic noise through a suppression or diminution of the shear layer instabilities and finally modifies the pressure distribution over the flap and the wing due to the morphing feedback. This leads to considerable modification on the aerodynamics loads that will be presented in the next section.

\section{B. Aerodynamic performances}

The morphing effects on the aerodynamic forces are carried out where the trailing-edge vibrate at different frequencies between $60 \mathrm{~Hz}$ and $400 \mathrm{~Hz}$ for an angle of incidence $\alpha=8^{\circ}$ for the same amplitude of actuation of the order $0.35 \mathrm{~mm}$

The Table. 1 presents the percentage of the change in the drag and the lift compared to the classical fixed wing-flap system. The amplitude of the load signals is increased due to the trailing-edge's dynamic vibration and its influence over the whole system, the signal becomes sinusoidal with a fixed period $T=1 / f_{a}$ that depends on the actuating frequency and the amplitude of the deformation. Calculating the mean values of the loads for each case, gave different results as presented in the Table. 1 due to the different generated smaller scales vortices which interact with the upper and lower flap and wing shear layers. Consequently, the actuation at $60 \mathrm{~Hz}$ revealed a reduction of the drag and a smaller reduction of the lift which causes an increase of the Lift/Drag ratio to $+1.53 \%$. In the other hand, it is shown that if the actuation frequency increases the drag and lift are also increased. But the drag is much more important than the lift, however these high frequencies of actuation could be more interesting for the landing flight stage, where the airplane needs more drag to diminish the speed. 


\section{Conclusion}

A two-dimensional OES and three-dimension OES-DDES numerical analysis of the dynamically morphed flap's trailing-edge was performed and the results are compared with different grids, in addition these results are quite similar to the $\mathrm{GA}(\mathrm{W})$ two-element configuration at $R e=2.2 \times 10^{6}$ a for a high angle of incidence $8^{\circ}$ and a flap deflection of $10^{\circ}$. VSI and TFI methods were implemented in the NSMB solver in order to impose the dynamic displacement of the sinusoidal trailing-edge movement. More reliable grid deformation was used in order to have accurate results and good mesh quality for the morphing configurations.

The numerical results of the modified A320 wing-flap when the actuation is activated at high frequency and low amplitude are quite similar to the published experimental results of the reduced scale single wing of the A320 prototype. It was found that the morphing generates new smaller eddies able to create an eddy blocking effect. This modifies the flow dynamics producing a thinner wake and a breakdown of bigger instabilities which leads to a decrease of the aerodynamic noise. Moreover, the signals of the aerodynamic forces are also impacted by the dynamic vibration, which results in some optimal regions of the morphing an increase of the mean lift/drag ratio compared to the static case.

For a future perspectives, we need to explore more the 3D numerical results in the morphing configuration and compare with the future results of the experimentation which are under tests in the IMFT wind tunnels.

\section{Acknowledgments}

The authors are grateful to the National Supercomputing centres CALMIP, CINES, HPC Strasbourg, IDRIS for the Computer allocation and the PRACE Supercomputing allocation $\mathrm{N}^{\circ}$ 2017174208. This work has been carried out within the Research programme N $^{\circ}$ 723402: "Smart Morphing and Sensing" (SMS) http://smartwing.org /SMS/EU. This project is funded from the European Union's H2020 program for research and development of technology.

\section{References}

[1] Chinaud, M., Rouchon, J., Duhayon, E., Scheller, J., Cazin, S., Marchal, M., and Braza, M., "Trailing-edge dynamics and morphing of a deformable flat plate at high Reynolds number by time-resolved PIV," Journal of Fluids and Structures, Vol. 47, 2014, pp. 41-54. doi:https://doi.org/10.1016/j.jfluidstructs.2014.02.007, URL http://www . sciencedirect . com/science/ article/pii/S0889974614000231, special Issue on Unsteady Separation in Fluid-Structure Interaction-1.

[2] Scheller, J., Chinaud, M., Rouchon, J., Duhayon, E., Cazin, S., Marchal, M., and Braza, M., “Trailing-edge dynamics of a morphing NACA0012 aileron at high Reynolds number by high-speed PIV," Journal of Fluids and Structures, Vol. 55, 2015, pp. 42-51. doi:https://doi.org/10.1016/j.jfluidstructs.2014.12.012, URL http://www. sciencedirect. com/science/ article/pii/S0889974615000158

[3] Chinaud, M., Scheller, J., Rouchon, J., Duhayon, E., and Braza, M., "Hybrid Electroactive Wings Morphing for Aeronautic Applications," Mechatronic Systems and Materials IV, Solid State Phenomena, Vol. 198, Trans Tech Publications, 2013, pp. 200-205. doi:10.4028/www.scientific.net/SSP.198.200.

[4] Jodin, G., Motta, V., Scheller, J., Duhayon, E., Döll, C., Rouchon, J., and Braza, M., "Dynamics of a hybrid morphing wing with active open loop vibrating trailing edge by time-resolved PIV and force measures," Journal of Fluids and Structures, Vol. 74, 2017, pp. 263-290. doi:https://doi.org/10.1016/j.jfluidstructs.2017.06.015, URL http://www. sciencedirect.com/ science/article/pii/S0889974616304121

[5] Nguyen, N., "Project Elastically Shaped Future Air Vehicle Concept NASA Innovation Fund Award,” Tech. rep., 2010.

[6] Urnes, J., Nguyen, N., C. Ippolito, J. T., Trinh, K., and E.Ting, A Mission Adaptive Variable Camber Flap Control System to Optimize High Lift and Cruise Lift to Drag Ratios of Future N+3 Transport Aircraft, 2013. URL https: //arc.aiaa.org/doi/abs/10.2514/6.2013-214

[7] Tô, J.-B., Simiriotis, N., Marouf, A., Szubert, D., Asproulias, I., Zilli, D., Hoarau, Y., Hunt, J., and M., "Effects of vibrating and deformed trailing edge of a morphing supercritical airfoil in transonic regime by numerical simulation at high Reynolds number," Journal of Fluids and Structures, 2019. doi:https://doi.org/10.1016/j.jfluidstructs.2019.02.011.

[8] Vos, J., Rizzi, A., Corjon, A., Chaput, E., and Soinne, E., Recent advances in aerodynamics inside the NSMB (Navier Stokes Multi Block) consortium, 1998. doi:10.2514/6.1998-225.

[9] LU, W., TIAN, Y., and LIU, P., "Aerodynamic optimization and mechanism design of flexible variable camber trailing-edge flap,” Chinese Journal of Aeronautics, Vol. 30, No. 3, 2017, pp. 988 - 1003. doi:https://doi.org/10.1016/j.cja.2017.03.003, URL http://wWw.sciencedirect.com/science/article/pii/S1000936117300754 
[10] Hoarau, Y., "Analyse physique par simulation numérique et modélisation des écoulements décollés instationnaires autour de surfaces portantes," Ph.D. thesis, 2002. URL http://www. theses. fr/2002INPTQ13H phD Thesis supervised by Marianna Braza, Fluid Dynamics, INP Toulouse, France 2002.

[11] Hoarau, Y., Pena, D., Vos, J. B., Charbonier, D., Gehri, A., Braza, M., Deloze, T., and Laurendeau, E., Recent Developments of the Navier Stokes Multi Block (NSMB) CFD solver, 2016. doi:10.2514/6.2016-2056.

[12] Martinat, G., Braza, M., Hoarau, Y., and Harran, G., "Turbulence modelling of the flow past a pitching NACA0012 airfoil at 105 and 106 Reynolds numbers," Journal of Fluids and Structures, Vol. 24, No. 8, 2008, pp. 1294 - 1303. doi:https://doi.org/10. 1016/j.jfluidstructs.2008.08.002, URL http://www. sciencedirect . com/science/article/pii/S0889974608000984. unsteady Separated Flows and their Control.

[13] Barbut, G., Braza, M., Hoarau, Y., Barakos, G., Sévrain, A., and Vos, J. B., "Prediction of Transonic Buffet around a Wing with Flap," Progress in Hybrid RANS-LES Modelling, edited by S. Peng, P. Doerffer, and W. Haase, Springer Berlin Heidelberg, Berlin, Heidelberg, 2010, pp. 191-204.

[14] Grossi, F., Braza, M., and Hoarau, Y., "Prediction of Transonic Buffet by Delayed Detached-Eddy Simulation," AIAA Journal, Vol. 52, No. 10, 2014, pp. 2300-2312. URL https://doi.org/10.2514/1.J052873.

[15] Braza, M., Perrin, R., and Hoarau, Y., "Turbulence properties in the cylinder wake at high Reynolds numbers," Journal of Fluids and Structures, Vol. 22, No. 6, 2006, pp. 757 - 771. doi:https://doi.org/10.1016/j.jfluidstructs.2006.04.021, bluff Body Wakes and Vortex-Induced Vibrations (BBVIV-4).

[16] Bourguet, R., Braza, M., Harran, G., and Akoury, R. E., "Anisotropic Organised Eddy Simulation for the prediction of non-equilibrium turbulent flows around bodies," Journal of Fluids and Structures, Vol. 24, No. 8, 2008, pp. 1240 - 1251. doi:https://doi.org/10.1016/j.jfluidstructs.2008.07.004, URL http://wwW.sciencedirect.com/science/article/pii/ S0889974608000820, unsteady Separated Flows and their Control.

[17] Spalart, P. R., "Detached-Eddy Simulation," Annual Review of Fluid Mechanics, Vol. 41, No. 1, 2009, pp. 181-202. URL https://doi.org/10.1146/annurev.fluid.010908.165130

[18] Guillaume, M., Gehri, A., Stephani, P., Vos, J., and Mandanis, G., "F/A-18 vertical buffeting calculations using unsteady fluid structure interaction," The Aeronautical Journal, Vol. 115, 2011, pp. 285-294. doi:https://doi.org/10.1017/S0001924000005777.

[19] Welch, P., "The use of fast Fourier transform for the estimation of power spectra: A method based on time averaging over short, modified periodograms," IEEE Transactions on Audio and Electroacoustics, Vol. 15, No. 2, 1967, pp. 70-73. doi:10.1109/TAU.1967.1161901.

[20] Jodin, G., Rouchon, J., Scheller, J., Simiriotis, N., Triantafyllou, M., Cazin, S., Elyakime, P., Marchal, M., and Braza, M., IUTAM Symposium on Critical flow dynamics involving moving/deformable structures with design applications, 2018.

[21] Hunt, J., Ishihara, T., Szubert, D., Asproulias, I., Hoarau, Y., and Braza, M., Advances in Fluid-Structure Interaction. Notes on Numerical Fluid Mechanics and Multidisciplinary Design, Vol. 133, 2016. 